\title{
A comparative study of sawtooth events and substorm onsets triggered by interplanetary shocks
}

\author{
Tushar Andriyas ${ }^{1, *}$ \\ ${ }^{1}$ DSK Post-Doctoral Fellow, Nehru Science Center, University of Allahabad, India - 211002
}

\author{
Article history \\ Received July 4, 2017; accepted November 20, 2017. \\ Subject classification: \\ Interplanetary Shocks; Geomagnetic Substorms; Sawteeth events; Auroral Oval.
}

\begin{abstract}
A comparison of solar wind conditions, geomagnetic response, and auroral boundary movement, using a similar number of sawtooth events and shock induced substorm triggers is carried out. $81 \%$ of the sawtooth onsets were triggered at low latitudes compared to $33 \%$ for onsets during shocks. Results of superposed epoch analysis indicated that the mean interplanetary magnetic field (IMF) $B_{z}$ remained strongly southward during sawtooth events while it was southward during the loading period and turned northward 20 minutes prior to the onset, during the shock triggered events. During both the event types, the mean energy available in the solar wind was found to be above the substorm threshold level. Relatively high levels of solar wind density indicated to the magnetosphere being compressed to a larger degree during substorms initiated by shocks. The high latitude indices were elevated during sawtooth events with 2$3 \mathrm{hr}$ fluctuations was observed in the AL and PCN indices but AU index after the onset remained at similar levels. SYM-H remained below storm time values during shock related onsets but was much stronger and remained above the storm threshold during sawtooth events, that they occurred embedded in a geomagnetic storm. Comparatively higher mean values of ASYM-H indicated to a much stronger asymmetric ring current during sawtooth events. Mean boundary locations during sawtooth events were located a few degrees equatorward of those during the shock related onsets. Oval in the dawn, dusk, and midnight sectors was much thicker during sawtooth events with clear widening around the onset during both event groups but the thickness in noon sector was similar and remained steady during the epoch window during both the event groups. Elevated driving during sawtooth events was also indicative in a greater stretching and relaxation of the magnetic field lines at geosynchronous orbit in the midnight sector.
\end{abstract}

\section{Introduction}

A substorm is a short lived disturbance with a life time of 2-4 hours [Tanskanen et al. 2002] in the magnetosphere-ionosphere coupled system that can deposit large quantity of energy into the auroral ionosphere. Substorms occur at time scales that are much faster when compared to a geomagnetic storm. Three phases have been ascertained as occurring during a substorm [McPherron et al. 1986]. The growth phase is generally initiated after the southward turning of solar wind interplanetary magnetic field (IMF) $B_{z}$. The expansion phase occurs when instabilities inherent to the magnetotail cause the diversion of the cross-tail current through the polar regions. This is recorded as a fluctuations in the local magnetic field that can reach levels above $1000 \mathrm{nT}$. The magnetosphere then returns to a steady state during the recovery phase. As such, substorms play an important role in energy circulation through the magnetosphere through dayside reconnection, energy storage in magnetotail, and release of this energy to relax the field lines to a more dipolar shape.

Interplanetary shocks (IPS) are plasma and magnetic field structures that travel through the solar wind and couple efficiently with planetary magnetospheres. Increase in the dynamic pressure associated with a shock structure leads to the compression of the magnetosphere indicated by large positive increases in the north-south (H) component of the geomagnetic field. If a geomagnetic storm occurs, such increases are termed as sudden impulses (SI) or storm sudden commencement (SSC) [Curto et al. 2007, Yao et al. 2009]. Sources of such structures lie in either coronal mass ejections (CMEs) or corotating interaction regions (CIRs) with the most of the shocks measured at Earth's orbit occurring due to CMEs. CIRs are flanked by forward and reverse shocks which only develop sufficiently after the Earth's orbit (1 AU) [Gosling and Pizzo 1999]. Shocks induce complex processes in the Earth's magnetosphere and as such are important in understanding the solar wind-magnetospheric coupling. Long periods of southward $B_{z}$ that precede an IPS can lead to sub- 
storm triggering and affect the amount of precipitated power [Liou et al. 1998].

IPS have been shown to trigger substorms and enhance nightside precipitation with powers reaching substorm levels [Meurant et al. 2005]. Zhou \& Tsurutani [2001] found that $43 \backslash \%$ of magnetic bays induced by shocks were associated with substorms. Using a global magnetohydrodynamic simulation, Oliveira and Raeder [2014] studied the effects of various types of IPS on the nightside magnetosphere. They observed that the frontal perpendicular shock led to a symmetric compression of the magnetotail to trigger a substorm and a large geomagnetic activity. Pressure pulses that accompany an IPS leads to compression of the magnetosphere on the dayside and can trigger substorms within minutes of arrival [Brittnacher et al. 2000]. During a period of southward IMF, an increase in the solar wind dynamic pressure can lead to the triggering of substorm expansion phase onset [Kokubun et al. 1977].

Sawteeth events have been classified as a separate class of activations that can occur during periods of moderately strong and steady solar wind [Pulkkinen et al. 2007]. Large quasiperiodic oscillations in the energetic particle fluxes, with a period of about $2-4 \mathrm{~h}$ [Henderson et al. 2006a], particularly in proton channels, at geosynchronous orbits were identified by Belian et al. [1995]. Rapid increase and slow decrease of the fluxes are a signature of such events. They generally occur during periods of increased solar wind driving with a continuous southward $B_{z}$. The saw blade like waveform is measured in both the energetic proton and electron fluxes. At geosynchronous orbit, the oscillation is observed as periodic stretching and relaxation of magnetic field lines [M. G. Henderson et al. 2006b]. This reconfiguration from a highly stretched to relaxed state has also been observed over a wide range of local time sectors [Pulkkinen et al. 2006]. These events also accompany a strongly enhanced partial ring current as measured by the ASY-H index.

Pulkkinen et al. [2007] statistically compared the solar wind conditions and geomagnetic activity during sawtooth and other substorm like electrojet storm time activations. They found that sawtooth events were characterized by a $2-3$ hour periodicity, strong stretching of the dusk sector field, and a strongly asymmetric ring current. The level of driving seen during sawtooth events was also found to be similar to that during other types of storm-time activations, the only difference being a weaker auroral (AL) response during sawtooth events. Partamies et al. (2009) statistically compared sawtooth events with periods of steady magnetospheric convections and isolated substorms. They reported that even though sawtooth events were strongly driven with a strong geomagnetic response, the coupling efficiency was the lowest. Solar wind speed also played a part in the type of geomagnetic activity with sawtooth events were triggered during times of elevated geomagnetic activity.

Given that the passage of IPS through the magnetosphere can lead to large increases in geomagnetic activity and that sawtooth events are found to occur during elevated solar wind driving, the similarities and differences between the two event types are investigated. In this paper, shock related substorm onsets and sawtooth events are compared with regards to the solar wind driving and the geomagnetic response. Superposed epoch analysis is used to quantify the similarities and differences between the two event groups using multiple in situ and ground based data. The datasets used in the analysis are discussed in section 2 . The results report on the superposed epoch analysis of the solar wind parameters in section $3 \mathrm{a}$, the ensuing geomagnetic activity at polar and equatorial latitudes in section $3 \mathrm{~b}$, and the auroral boundary movements with the associated magnetic field conditions at geosynchronous orbit in section $3 \mathrm{c}$. The results of the analysis are discussed further with previous literature in section 4 and the conclusions from the study are presented in section 5 .

\section{Data and Procedure}

The solar wind and the subsequent geomagnetic response data at 1 minute ( $\mathrm{min}$ ) cadence, was obtained from http://cdaweb.gsfc.nasa.gov/ [King and Papitashvili 2005], during the years 2000 to 2002. In this study, IMF $B_{x}, B_{y}$, and $B_{z}$ in $\mathrm{nT}$ (in Geocentric Solar Magnetospheric or GSM coordinates), solar wind flow speed (Vx in $\mathrm{km} / \mathrm{s}$ ), and plasma ion density, $N_{p}$ in particles $\mathrm{cm}^{-3}$ were used. Derived parameters such as the flow pressure, $P(\mathrm{nPa})$, the IMF magnitude, $B_{\text {tot }}=\sqrt{ } B_{x}{ }^{2}+B_{y}{ }^{2}+B_{z}{ }^{2}$ (nT), solar wind electric field $\left(E_{y}\right.$ in $\left.\mathrm{mV} / \mathrm{m}\right)$, and the epsilon parameter (in TW), $\Theta=\tan ^{-1}\left(B_{y} / B_{z}\right.$ ) being the solar wind clock angle, were calculated from the primary parameters. Magnetospheric response was measured through $A L, A U, A E$ (all in $\mathrm{nT}$ ), $\mathrm{PCN}(\mathrm{mV} / \mathrm{m})$, SYM, and ASYM indices (nT). Substorm onset timings and locations were derived from the Frey et al. [2004] database.

Sawteeth events were searched for in a list compiled by R. L. McPherron, who identified the events visually from the particle injections as measured by the Los Alamos Synchronous Orbit Particle Analyzer (SOPA) onboard the geostationary 1990-095,1991-080, 1994-084, LANL-97A, LANL-01A, and LANL-02A satel- 
lites at multiple magnetic local time sectors [Pulkkinen et al. 2007]. The shock parameters were calculated based on high resolution solar wind parameters (as obtained from Solar Wind Electron Proton Alpha Monitor (SWEPAM) instrument [McComas et al. 1998] and the IMF from the MAGnetic field experiment (MAG) [Smith et al. 1998] instrument, on board the Advanced Composition Explorer (ACE) satellite). The time of the shock interface as measured by the ACE satellite was checked with the Harvard-Smithsonian shock database located at https://www.cfa.harvard.edu/shocks/.

Rankine-Hugonoit equations of conservation were used to determine the speed of the shock along the normal direction [Oliveira 2017]. This unit normal vector was calculated based on the Abraham-Shrauner mixed-mode analysis technique [Abraham-Shrauner 1972] as given by the following equation:

$$
\hat{n}=\frac{\left(\vec{B}_{2}-\vec{B}_{1}\right) \times\left(\left(\vec{V}_{2}-\vec{V}_{1}\right) \times \vec{B}_{2}\right)}{\left|\left(\vec{B}_{2}-\vec{B}_{1}\right) \times\left(\left(\vec{V}_{2}-\vec{V}_{1}\right) \times \vec{B}_{2}\right)\right|}
$$

with subscripts 1 and 2 indicating upstream and downstream values and $\vec{B}$ and $\vec{V}$ representing the solar wind magnetic field and velocity, respectively. Shock speed, $V_{s}$, was calculated using the expression [Tsurutani and Lin 1985]:

$$
V_{S}=\left(\frac{N_{2} \vec{V}_{2}-N_{1} \vec{V}_{1}}{N_{2}-N_{1}}\right) \cdot \hat{n}
$$

$\mathrm{N}$ being the plasma density. After calculating the angle $\theta$ between the upstream magnetic field and the shock normal, the other parameters such as magnetosonic speed and Mach number can be calculated as

$$
\begin{gathered}
V_{m s}=\sqrt{\frac{\left(V_{A}^{2}+C_{S}^{2}\right) \pm \sqrt{\left(V_{A}^{2}+C_{S}^{2}\right)^{2}-4 C_{S}^{2} V_{A}^{2} \cos ^{2}}}{2}} \\
M_{m s}=\frac{V_{s}}{V_{m s}}
\end{gathered}
$$

where $V_{A}$ and $C_{S}$ represent the upstream Alfvenic and sound speeds, respectively.

Poleward and equatorward boundary data used to study the oval movement during the onsets was obtained from the British Antarctic Survey (BAS). These boundaries were obtained from the images obtained Imager for Magnetopause-to-Aurora Global Exploration (IMAGE) satellite as described in Longden et al. [2010]. Single and double Gaussians were used to fit to the average intensity profiles in each of the 24 magnetic local time (MLT) bins. Non-linear least square fitting yielded parameters that were then used to estimate the equatorward and poleward luminosity boundaries in each MLT sector.
The auroral oval boundary locations were further averaged into MLT bins based on the noon, dusk, midnight, dawn sectors. In this study, the noon sector was chosen to be between 1000-1500 MLTs, dusk between 1600-2100 MLTs, midnight sector between 2000-0300 MLTs [Sandholt et al. 2014], and dawn sector between 0400-0900 MLTs, respectively. Additionally, magnetic field at geosynchronous orbit as measured by GOES 8 and 10 (active during the years 2000-2002) magnetometers (Hp component in the local spacecraft frame), was used to evaluate the amount of magnetotail stretching and relaxation around the substorm onsets [Singer et al. 1996]. Data from the two satellites were used when they were within the MLTs around the midnight sector.

\section{Results}

The time at which a shock interface was measured was compared against the substorm onset from the database provided by Frey et al. [2004] that occurred in a window of 120 mins after the shock. During the years 2000-2002, 58 unique shocks were observed prior to a substorm onset that could have triggered a substorm. All the shocks measured were found to be fast forward shocks. During the same years, 63 unique sawtooth events had onsets that matched with the Frey et al. [2004] onset list. The shocks were either of the fast forward or fast reverse type, with the majority (55) of the onsets triggered by fast forward type shocks. Within the shocks, the mean magnetic compression was around 2 while the plasma was compressed by a factor of around 2.3. A mean shock angle (of the magnetic field w.r.t. the shock normal) of 610 indicated that, on average, the shocks were oblique.

The onset location (between 50-90o) in the altitude-adjusted corrected geomagnetic coordinates or AAGCM [K. B. Baker and Wing 1989], for both the event classes were plotted. In Figure 1, the magnetic latitude and MLT of the onsets associated with shocks and sawtooth events are plotted in blue and red, respectively. As seen in the figure, the onset locations have a preference for premidnight sector. It can be noted that majority $(81 \%)$ of the sawtooth events occur at low latitudes (below 630 ) while only $33 \%$ of the shock initiated onsets are triggered at such low latitudes. This should indicate a high solar wind driving associated with sawtooth events as measured by the electrojet indices [Grocott et al. 2009]. A superposed epoch analysis was conducted during a time window of 720 mins around the onset with the reported superposed epoch curves being the mean of the individual events, respectively. 


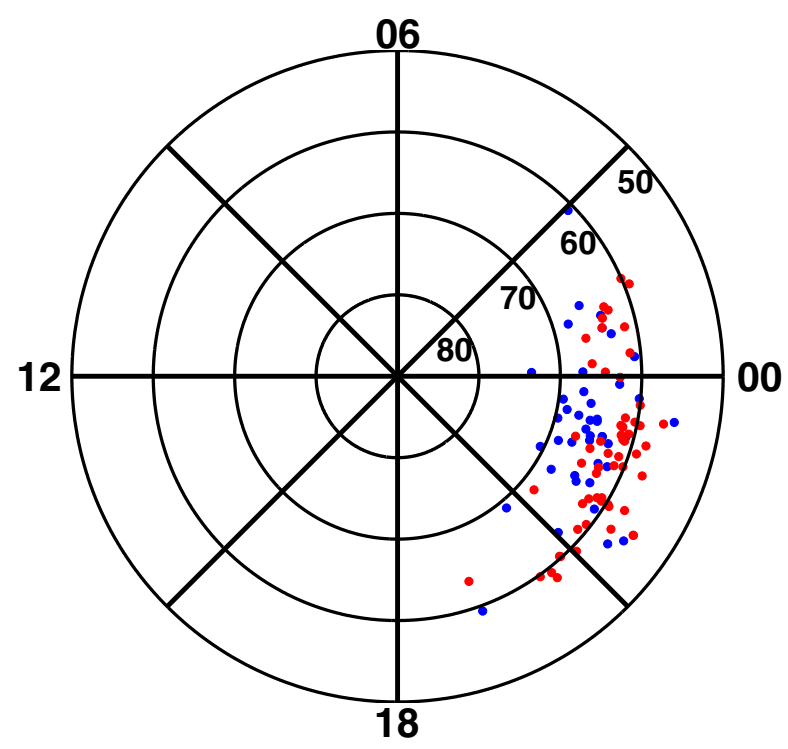

Figure 1. Substorm onset locations associated with the 58 shock and 63 sawtooth events indicated by blue and red filled circles. Midnight (00 hrs), dawn (06 hrs), day (12 hrs), and dusk (18 hrs) MLTs are indicated along with the magnetic latitude.

\subsection{Mean solar wind conditions}

Superposed epoch analysis is used in this section to study the similarities and differences between the two event types (blue curves for shocks and red for sawtooth events) with regards to the solar wind driving and the geomagnetic response. The mean IMF curves resulting from the superposed epoch analysis are shown in Figure 2. The values are plotted over an epoch window of 720 mins around the substorm onset (vertical black dashed line) as obtained from the Frey et al. [2004] list. The mean IMF $B_{x}, B_{y}, B_{z}$, and $B_{\text {tot }}$ are plotted in blue for shocks and in red line for sawtooth events. The mean $B_{x}$ and $B_{y}$ do not exhibit any significant changes during the epoch window with a preference for a zero average value for most of the epoch window, during shock related onsets. However, the mean $\mathrm{Bx}$ and By during sawtooth events exhibit a Parker like sector structure with the IMF pointing away from the Sun [Partamies et al. 2009].

Mean $B_{z}$ for the shocks turns southward from a zero average value to around $-6 \mathrm{nT}$ around the substorm onset and turns northward around the onset, which may be indicative of magnetotail loading/unloading [D. N. Baker et al. 1996]. The total IMF strength becomes stronger around 100 mins and strongest 20 mins prior to the onset related to shocks. During sawtooth events, the mean $B_{z}$ remains negative throughout the epoch window $(\sim-4 \mathrm{nT})$ and starts to decrease to more negative values 120 mins prior to the onset. It can be seen that there is a reduction in the mean $B_{z}$ values to around $-7 \mathrm{nT}, 20$ mins prior to the substorm onset and a return to less negative values post onset. However, during the sawtooth events, the total field remains much stronger (double) compared to that for the shock related onsets outside a window \pm 100 mins around the onset.

The mean solar wind plasma parameters are plotted in Figure 3. The shocks on an average compressed the solar wind by a factor of two, with the density increasing from around 6 particles $\mathrm{cm}^{-3}$ to 13 particles $\mathrm{cm}^{-3}$ around the onset and remained around 10 particles $\mathrm{cm}^{-3}$ through the epoch window after the onset.

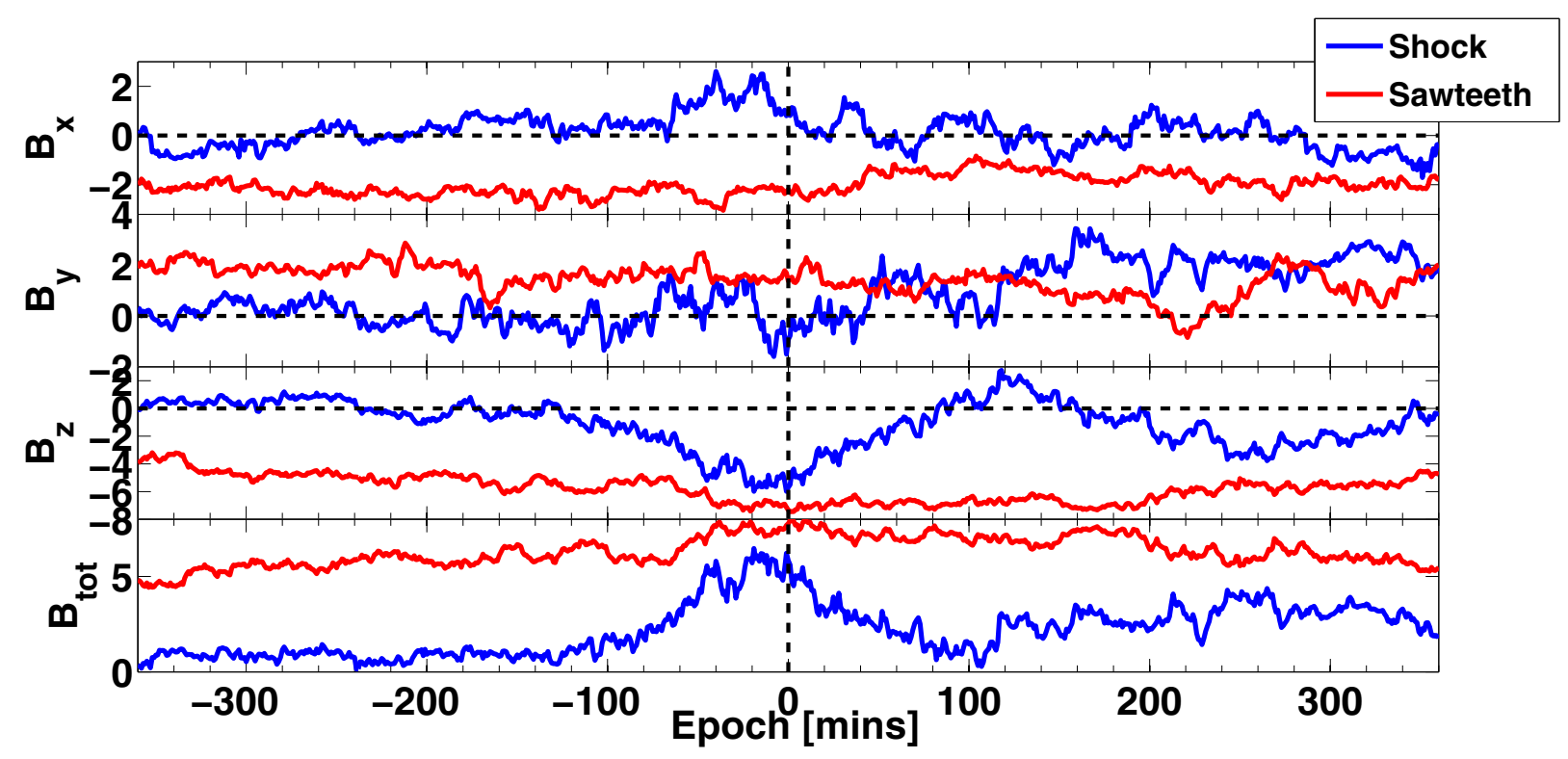

Figure 2. Mean IMF components with $B_{x}, B_{y}, B_{z}$, and the magnitude of the IMF, $B_{\text {tot }}$ plotted in blue and red lines for the shocks and sawtooth events, respectively, over an epoch window of 720 minutes around the substorm onset (zero epoch). The vertical and horizontal dashed black lines indicate the zero mean and zero epoch, respectively. 


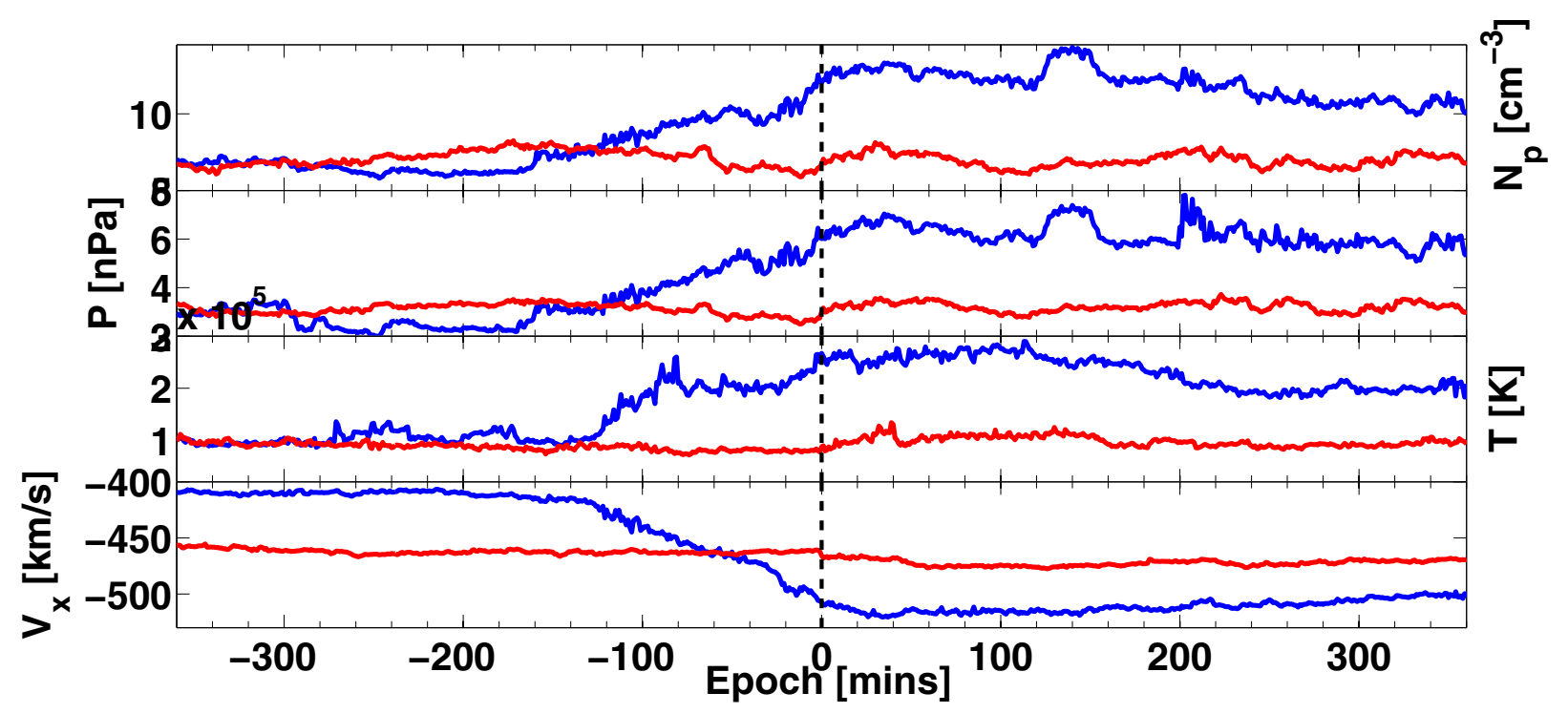

Figure 3. Mean solar wind plasma parameters for the shock (blue) and sawtooth (red) events. Mean density (particles $\mathrm{cm}^{-3}$ ), dynamic pressure $(\mathrm{nPa})$, temperature $(\mathrm{K})$, and solar wind velocity $\left(V_{x} \mathrm{~km} / \mathrm{s}\right)$ are plotted from top to bottom.

The mean dynamic pressure, being a function of both the solar wind velocity and density, also increased from around $2 \mathrm{nPa}$ to $7 \mathrm{nPa}$ around the onset and remained steady at around $6 \mathrm{nPa}$ post onset. Elevated mean temperature prior to the substorm onset as a result of shock compression can be seen with average temperature increasing from around $1 \times 10^{5} \mathrm{~K}$ to around $2.5 \times 10^{5}$ $\mathrm{K}$ around the onset. During the sawtooth events, mean $N_{p}$ remained steady at around 6-8 particles $\mathrm{cm}^{-3}$ with a mean dynamic pressure around $3 \mathrm{nPa}$ and a mean temperature of around $1 \times 10^{5} \mathrm{~K}$. For the shock related onsets, the mean solar wind velocity, $V_{x}$, increased across the shock interface before the substorm onset. The mean velocity became faster around the substorm onset from around $-410 \mathrm{~km} / \mathrm{s}$ prior to and $-520 \mathrm{~km} / \mathrm{s}$ after the substorm onset. During the sawtooth events, it increased marginally from $-460 \mathrm{~km} / \mathrm{s}$ to around -480 $\mathrm{km} / \mathrm{s}$ after the onset.

During both the event groups, the epsilon parameter lies below $300 \mathrm{GW}$, with the shock related onsets experiencing a large increase in the mean epsilon parameter, 90 mins prior to the substorm onset (upper panel in Figure 4). As indicated in Figure 2, mean IMF $B_{z}$ turns southward 90 mins prior to the onset during shock related onsets. As a result, this increased $B_{z}$ contributes to an increased value of the mean epsilon. Dur-

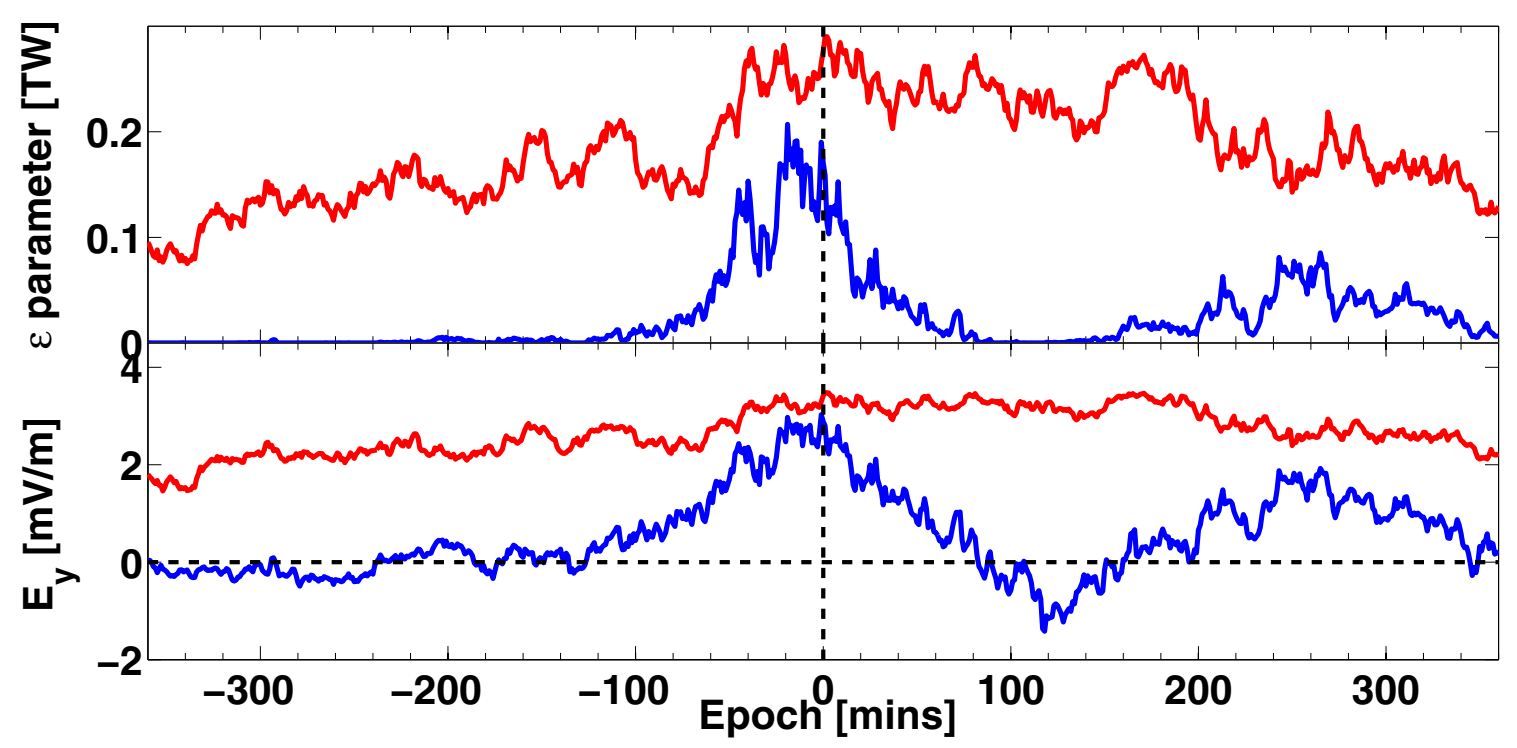

Figure 4. Mean epsilon parameter (TW) and solar wind electric field $(\mathrm{mV} / \mathrm{m})$ in the dawn-dusk direction for the 58 shock (blue) and 63 sawtooth (red) events. 


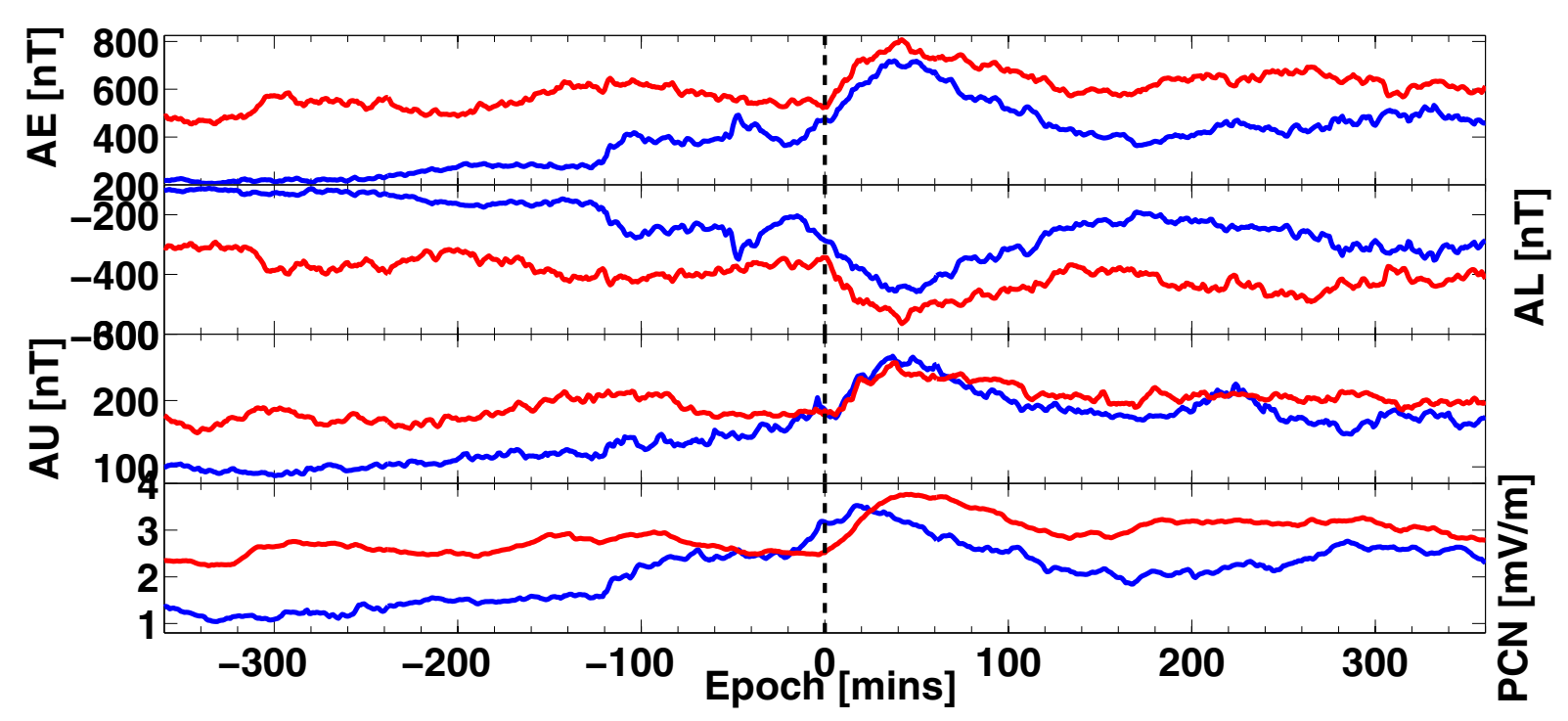

Figure 5. Mean high latitude geomagnetic response as measured by the $A E, A L, A U$, and PCN indices (top to bottom) for the shock related (blue) and sawtooth (red) events.

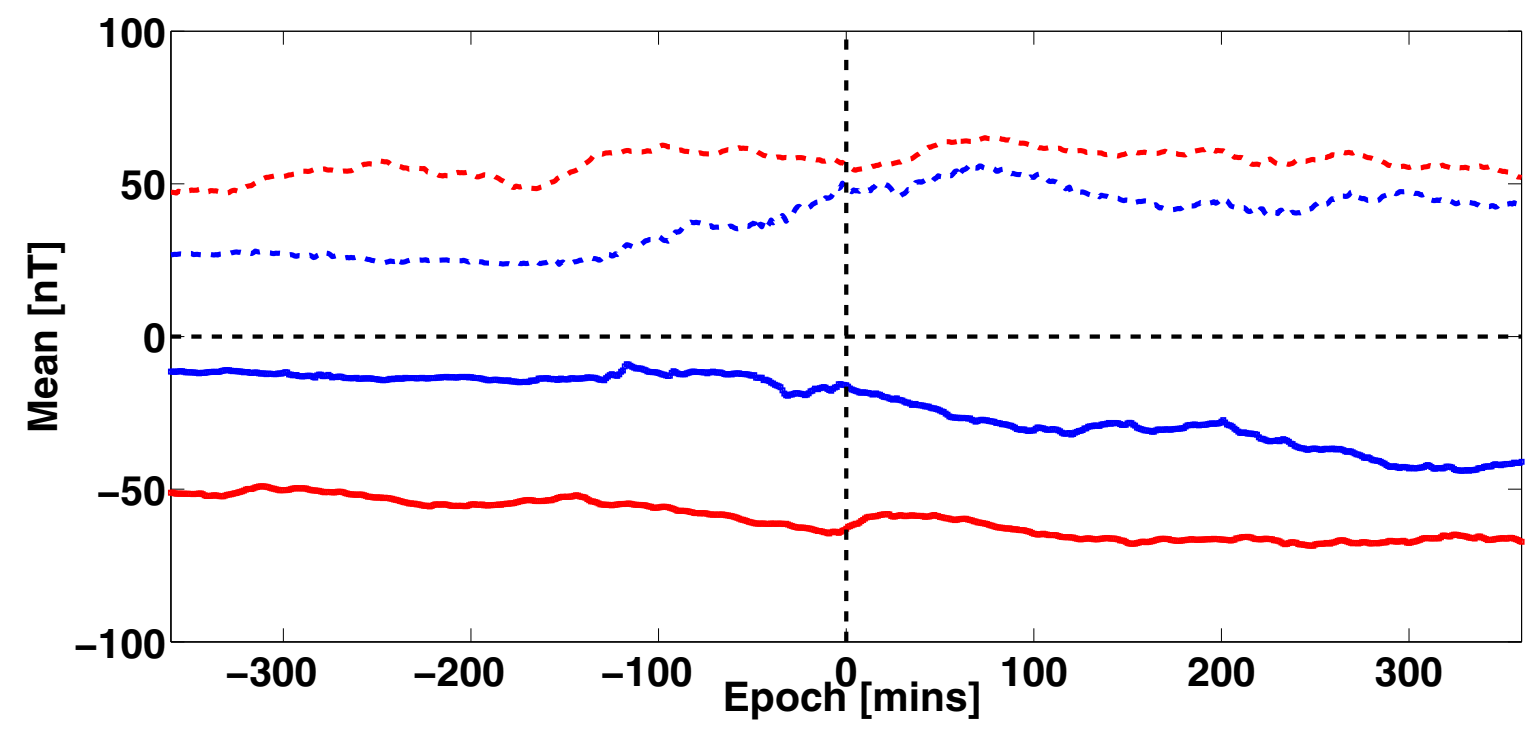

Figure 6. Mean SYM-H (continuous lines) and ASYM-H (dotted lines) indices during the shock related onsets (indicated in blue) and sawtooth events (indicated in red).

ing the sawtooth events, a steady southward IMF $B_{z}$ implies that the mean epsilon parameter remains elevated throughout the epoch window. The mean epsilon parameter increases to values around $200 \mathrm{GW}$ close to the substorm onset for the shock related events while it remains between 100-200 GW before increasing to values close to $300 \mathrm{GW}$ around the onset for the sawtooth events. These values are above the substorm loading threshold of $100 \mathrm{GW}$ [Akasofu 1981]. However, in both the cases the storm threshold of $1 \mathrm{TW}$ is not exceeded.

For the shock related onsets, an increase in both the mean solar wind velocity and more negative $B_{z}$ translated to an increase in the solar wind electric field $E_{y}=V_{x} B_{z}$ ), plotted in blue in Figure 4. The electric field recovered to preonset values around 80 mins after the onset as seen in the figure. Compared to shocks related onsets, the mean electric field during sawtooth events remained elevated throughout the epoch window at values between 1-2 $\mathrm{mV} / \mathrm{m}$ with a slight increase close to the onset owing to an increase in the solar wind speed and $B_{z}$ becoming more negative.

\subsection{Magnetospheric response}

Mean geomagnetic activity at high latitudes as measured by the $A E, A L, A U$, and PCN indices, are plotted in Figure 5. Sawteeth events produce a stronger response in the indices the mean $A E, A L, A U$, and PCN reaching peak values close to $800 \mathrm{nT},-600$ 
$\mathrm{nT}, 250 \mathrm{nT}$, and $4 \mathrm{mV} / \mathrm{m}$, when compared to those for the onsets driven by shocks (700 nT, $-450 \mathrm{nT}, 250 \mathrm{nT}$, and $3.5 \mathrm{mV} / \mathrm{m}$ ). Expansion phase can be seen to occur for almost the same duration for both the event types (100 mins). A recovery to pre onset values can be seen 120 mins after the onset for both the classes. The mean values of $A L$ and as a result $A E$ indices remain elevated during the entire epoch window during the sawtooth events. $A U$ index, on the other hand, experiences an almost equal increase after the onset during the two event groups. PCN increases by $1.5 \mathrm{mV} / \mathrm{m}$ for the sawtooth events compared to $1 \mathrm{mV} / \mathrm{m}$ for the shock related onsets.

Equatorial activity indices follow the same trend as for the high latitude indices, with much stronger response to the sawtooth related solar wind driving as seen in Figure 6. Shock related onsets lead to a decrease in the mean SYM-H of around $-40 \mathrm{nT}$ where during the sawtooth events, the index dips to values close to $-70 \mathrm{nT}$ and remains elevated throughout the epoch window. Variations in the mean ASYM-H index are seen to be more quasi-periodic and fairly steady during the sawtooth events compared to the shock related onsets, during which the ASYM-H experiences a sudden increase from values around $25 \mathrm{nT}$ to $55 \mathrm{nT}$ around the onset as seen in Figure 6. Higher mean values of ASYM-H during sawtooth events indicate to a much stronger asymmetric ring current compared to that during shock related onsets.

\subsection{Auroral oval and field line stretching}

As indicated in section 2, the auroral oval boundaries were averaged into MLT bins based on the dayside, dusk, midnight, dawn sectors to investigate the differences in auroral movement between the two event groups. Figure $7 \mathrm{a}$ are plots of the mean equatorward and poleward boundaries in the four sectors, while the plots in Figure $7 \mathrm{~b}$ represent the respective mean oval thickness in the sectors. Both the mean boundaries during the sawtooth events are located equatorward of the oval boundaries during shock initiated onsets indicating a stronger stretching of the field lines during sawtooth events. Before the substorm onset, an equatorward descent in both the boundaries can be seen in the sectors, with the poleward boundary moving faster than the equatorward boundary in any given sector. A sudden equatorward descent is observed in the mean equatorward boundaries during the shock related onsets, especially in the dawn, dusk, and midnight sectors. Due to stronger solar wind forcing during sawtooth events, this equatorward advancement in the mean equatorward boundaries is barely noticeable in the already strongly stretched field lines.

The top left panel in Figure $7 \mathrm{a}$ indicates that in the dusk sector (1600-2100 MLT), the mean equatorward boundary starts to retreat equatorward around 40 mins prior to the onset, during the shock induced onsets (blue continuous line) compared to the sawtooth events, during which the boundary gradually moved equatorwards. The mean boundary recovers to preonset latitudes during sawtooth events but remains at lower latitudes for the shock related onsets. Clear preonset equatorward departure and poleward descent after the onset can be seen in the mean poleward boundary for the sawtooth events and is observed to be much stronger compared to shock related onsets. In the noon sector (1000-1500 MLT), both the mean equatorward and poleward boundaries tend to move equatorward at the same rate after the onset, as seen in the top right panel of Figure $7 \mathrm{a}$. This trend is similar for both sawtooth events and shock related onsets with the only difference being that the boundaries during the sawtooth events are located equatorward of the mean oval boundaries during the shock related onsets.

A similar trend is seen in the mean oval boundaries around the dawn sector (0400-0900 MLT), with equatorward advancement prior to the onset and poleward retreat after the substorm onset. Equatorward movement is seen to be much weaker in the equatorward boundary but strong relaxation can be seen in the poleward retreat of the poleward boundary, during the sawtooth events. Conversely, the mean equatorward boundary experiences a much stronger equatorward descent in the equatorward boundary and slight variations in the poleward boundary location during the shock related onsets. Mean boundaries in the midnight sector (2000-0300 MLT) for the two event groups are plotted in the bottom right panel of Figure $7 \mathrm{a}$, which can be seen to have the most dynamics. Equatorward movement of the boundaries can be seen prior to the onset caused by the stretching of field lines in the magnetotail. After the onset, the equatorward boundaries gradually move poleward for both the event groups while the poleward departure of the poleward boundaries occurs at a comparatively faster rate. After the onset, the poleward boundary also experienced a large poleward retreat from around $68 \mathrm{o}$ to 730,30 mins after the onset during sawtooth events while a change from 70 oto 730 was observed in 15 mins during shock related onsets. However, during the shock related onsets, the poleward boundary could not recover to its preonset location for the remaining epoch. 

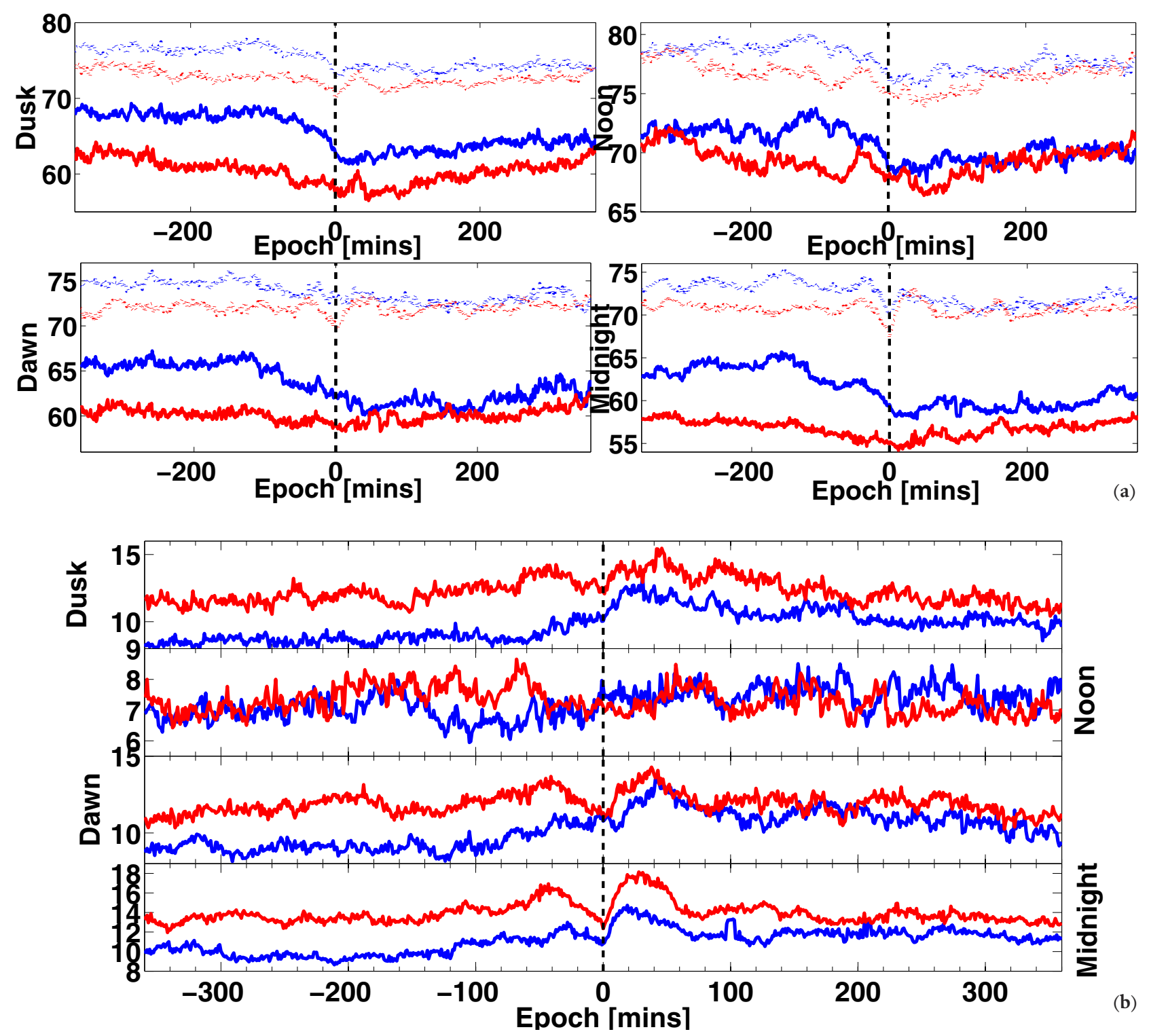

Figure 7. Mean oval boundaries (continuous lines for equatorward locations and dotted lines for poleward locations) during sawtooth events (red) and shock triggered (blue) onsets in Figure $7 \mathrm{a}$ in the four oval sectors as discussed in the text. Figure $7 \mathrm{~b}$ is the mean oval thickness during the two event groups.

The mean oval thickness (Figure $7 \mathrm{~b}$ ) during the sawtooth events is observed to be greater than during the shock related onsets in the dawn, dusk, and midnight sectors. The mean oval around the dusk sector can be seen to be much thicker for the sawtooth events, but the oval around the onset time is more dynamic during the shock related onsets. Given that both the respective mean boundaries in both the event groups moved at the same rate in the noon sector, the mean oval thickness was around the same for both the event types with the mean width fluctuating between 6-80 latitude. Clear thinning prior to the onset can be seen during the sawtooth events which is absent during the shock related onsets in the dawn sector. Rapid widening of the oval can be seen in the dawn sector after the onset compared to the dusk sector, in both the event groups. This thinning prior to the onset and widening post onset for the event groups is attributed due to fast poleward retreat of the mean poleward boundary combined with a relatively gradual poleward retreat of the equatorward boundary. Rapid thinning before and widening after the onset can be seen in the oval thickness in the midnight sector during both the event groups. This process is more pronounced for the sawtooth events due to the poleward boundary changing rapidly around the onset (Figure 7a) in the midnight sector.

In order to study the magnetic field in the midnight sector at geosynchronous orbit during substorm onsets [Singer et al. 1996], superposed means for the magnetic field, $H_{p}$ (in the local spacecraft coordinates, measured in nT) were generated for GOES 8 (blue and red continuous lines for shocks and sawtooth events, 
respectively) and 10 (blue and red dashed lines for shocks and sawtooth events, respectively). Field strength weakening prior to the onset led to the equatorward descent of both the mean boundaries, as noted in Figure 7a. Mean field values increased after the onset, indicative of a poleward retreat of the boundaries. The mean stretching and relaxation signature is weaker for the onsets triggered by shocks compared to those during sawtooth events. The mean poleward and equatorward boundaries during sawtooth events were found to be equatorward of those found during shock triggered substorms. As seen in Figure 8, the mean $H_{p}$ at GOES 8 and 10 location was found to be much stronger (dipolar) during shock related onsets as indicated by values between 85 and $95 \mathrm{nT}$. Weak stretching prior to the onset (to values around $80 \mathrm{nT}$ ) and relaxation post onset (to values close to $90 \mathrm{nT}$ ) was observed in the mean $H_{p}$ during shock related onsets. Comparatively, during sawtooth events, the magnetic field was much weaker (or stretched more) prior to the onset with a much stronger relaxation at both GOES 8 and 10 locations. This is consistent with the movement of mean poleward boundary during the sawtooth events in the midnight sector (bottom right panel of Figure 7a), which retreated poleward to a much greater degree when compared to the onsets triggered by shocks.

\section{Discussion}

The response of Earth's magnetosphere to the solar wind driving during substorm onsets related to shocks and sawtooth oscillations is investigated. With almost equal number of events ( 58 shock related triggers and 63 sawtooth events) in the two groups, a su- perposed epoch analysis was performed based on merged OMNI data and GOES observations, during 2000-2002. It was found that around $81 \%$ of the sawtooth events and $33 \%$ of shock driven triggers were at latitudes below 63o latitude. Previously, Grocott et al. [2009] had reported that onsets at low latitudes lead to intense auroral activity, and a similar observation was made in the case of sawtooth events. Superposed epoch analysis was performed on these event groups with onset timing derived from Frey et al. [2004], over an epoch window of 720 mins.

Mean negative values of IMF $B_{x}$ and positive values of IMF By during sawtooth events indicated to a sector structure with IMF pointing away from the Sun in the equatorial plane. Comparatively, during shock related onsets, the mean IMF $B_{x}$ and $B_{y}$ did not have any preference for either positive or negative values and averaged to zero over the epoch window. Mean IMF $B_{z}$ remained strongly negative during sawtooth events (stronger than that during the shock related onsets) throughout the epoch window while it turned southward around a 100 mins before the onset and turned northward 20 mins before the onset. The total field strength was also found to be much stronger for sawtooth events compared to the shock related triggers.

For shocks that occurred in a window of 120 mins prior to the onset, the mean velocity jumped from about 400 to $600 \mathrm{~km} / \mathrm{s}$. This was accompanied by jumps in particle density (from around 6 to 13 particles $\mathrm{cm}^{-3}$ ) and a jump in the mean dynamic pressure from 2 to around $7 \mathrm{nPa}$ around the onset. The mean solar wind values during sawtooth events were stable around $460 \mathrm{~km} / \mathrm{s}, 7$ particles $\mathrm{cm}^{-3}$, and $3 \mathrm{nPa}$ during the whole

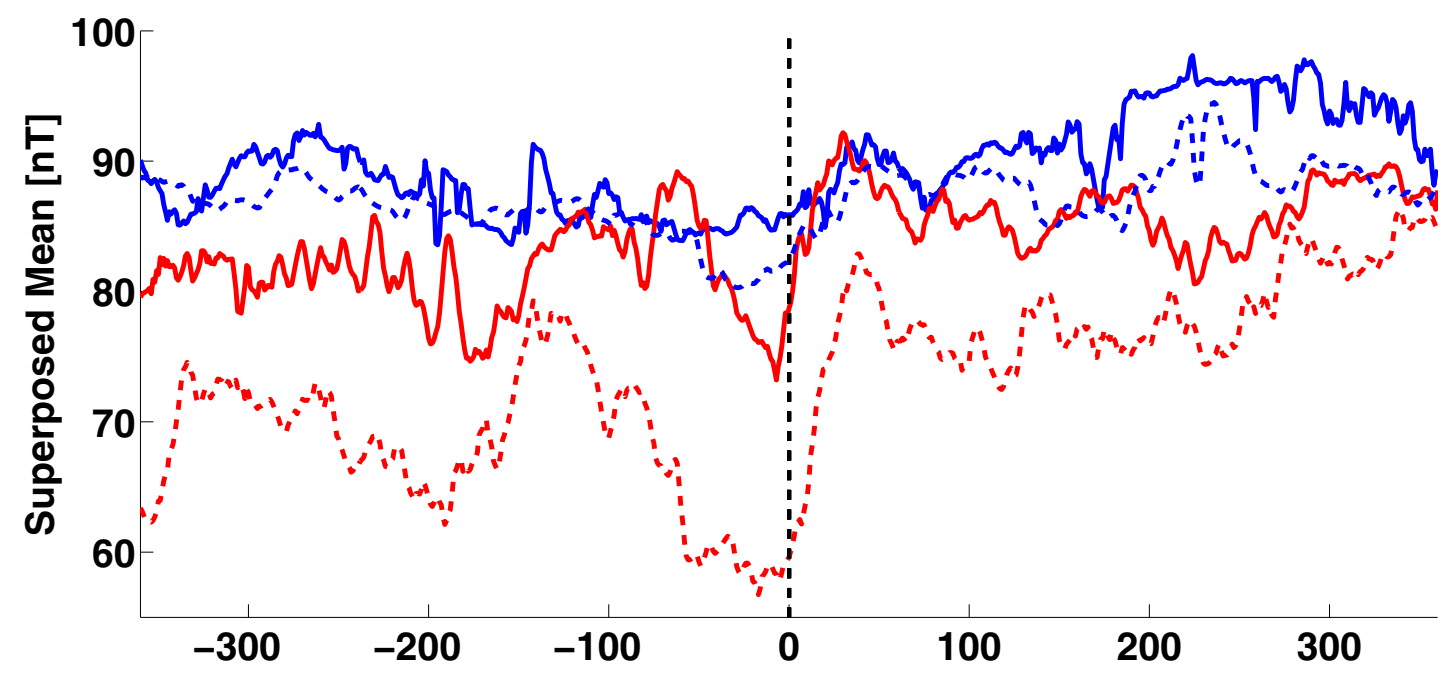

Figure 8. Mean GOES 8 (continuous lines) and 10 (dashed lines) Hp magnetic field during the two event groups. Red color indicates the mean values during sawtooth events while plots in blue indicate the mean Hp values during shock related onsets. 
epoch window. The dayside compression was therefore, much stronger during shock triggered onsets.

The mean epsilon parameter increased to values around $200 \mathrm{GW}$ around the substorm onset for the shock related events while it remained between 100$200 \mathrm{GW}$ before increasing to values close to $300 \mathrm{GW}$ around the onset for the sawtooth events. These values are above the substorm loading threshold of 100 GW [Akasofu 1981]. However, in both the cases the storm threshold of $1 \mathrm{TW}$ was not exceeded. Mean $E_{y}$ close to $3.5 \mathrm{mV} / \mathrm{m}$ around the onset and elevated values during the whole epoch indicated to the sawtooth events being driven strongly. Relatively, during the shock related triggers, the mean $E_{y}$ reached peak values close to $2.5 \mathrm{mV} / \mathrm{m}$ and did not remain at these values given the nature of mean IMF $B_{z}$ which turned northward 20 mins prior to the onset.

High latitude indices were also found to be elevated during sawtooth events compared to shock related triggers. Given that majority of the sawtooth onsets were triggered at latitudes below 630, they were associated with a strong electrojet response as is the case with low latitude substorm onsets. Mean $A L$ index reduced by almost $100 \mathrm{nT}$ more during sawtooth events compared to shock related triggers. Although the $A U$ index was lower prior to the onset, during shock related triggers, the mean values reached the same levels as found during sawtooth events. This difference in behavior of the $A L$ and $A U$ indices could be attributed to how $A U$ and $A L$ indices describe the eastward and westward electrojets [Kamide and Kokubun 1996, Kamide and Rostoker 2004]. This was also the case with the PCN index with values during both event groups reaching peak values around $3.5-3.8 \mathrm{mV} / \mathrm{m}$. However, these peak values were reached 20 and 50 mins after the onset during shock related and sawtooth events, respectively. Both $A L$ and PCN indices indicated to a 2-3 hour (hr) periodicity as also reported in Pulkkinen et al. [2007].

Sawteeth events occurring usually during geomagnetic storms (Dst $\leq-50 \mathrm{nT}$ ) [Kallio et al. 2000, Partamies et al. 2009], can be seen in comparatively large values of the SYM-H index. The quasi-periodic fluctuations during sawtooth events in the high latitude indices was also seen as the partial recovery of the SYM-H index corresponding to the individual teeth [M. G. Henderson et al. 2006]. Large quasi-periodic variations, specially prior to the onset were observed in the ASYM-H index during sawtooth events. The initial decrease and increase in ASYM-H response to quasiperiodic flux injections at geosynchronous orbit was delayed by around 40 mins compared to that in the
SYM-H index [Pulkkinen et al. 2006].

Stronger mean solar wind driving within sawtooth events was also seen in the oval boundary locations and dynamics. The mean auroral boundaries during sawtooth events were found to be located equatorward in all the sectors when compared to the boundary locations during shock triggered substorms. This indicated that the magnetosphere was stretched to a greater degree during sawtooth events (as also seen in the strength of geosynchronous magnetic field measured by the GOES satellites) and that the oval was thicker by at least $2-4 \mathrm{O}$ in the dawn, dusk, and midnight sectors. The poleward retreat of the mean poleward boundary was much stronger during the sawtooth events in the dawn and midnight sectors. It was observed that the both the mean equatorward and poleward boundaries in the day sector proceeded equatorward at the same rate around the onset and this meant that the the oval thickness during the two event groups was almost the same.

Equatorward movement of the boundaries seen prior to the onset caused by the stretching of field lines in the magnetotail during the growth phase of a substorm [Kozelova and Kozelov 2013]. As seen in Figure 8 , the mean field is much weaker (stretching) during sawtooth events compared to shock related onsets as also indicated in Figure $7 \mathrm{a}$, where the oval boundaries, during sawtooth events, were located equatorward of the boundaries during shock related onsets. This is a result of much stronger and persistent southward IMF $B_{z}$ during sawtooth events. During sawtooth events, the magnetic field was much weaker (or stretched more) prior to the onset with a much stronger relaxation at both GOES 8 and 10 locations. This is consistent with the movement of mean poleward boundary during the sawtooth events in the midnight sector (bottom right panel of Figure 7a), which retreated poleward to a much greater degree when compared to the onsets triggered by shocks.

\section{Conclusions}

As shocks lead to elevated geomagnetic activity and sawtooth events are found to occur during elevated solar wind driving, a comparative study between sawtooth events and substorm onsets triggered by shocks was carried out. Superposed epoch analysis was used to examine the similarities and differences between 58 shock related onsets and 63 sawtooth events during the years 2000-2002.

- During both the event types, the mean energy available in the solar wind (as determined by the epsilon parameter) was found to be above the 
substorm threshold level of $100 \mathrm{GW}$ [Akasofu 1981]. During sawtooth events, the mean epsilon parameter was around $300 \mathrm{GW}$ around the onset compared to $200 \mathrm{GW}$ during shock related onsets. The sudden increase in mean epsilon parameter around the onset during shock related events indicates to a result previously found by Akasofu and Chao [1980], who indicated that a substorm could not only be triggered by a storm sudden commencement but was a direct consequence of increased power of the solar windmagnetosphere dynamo.

- Both the high latitude indices were elevated during sawtooth events with 2-3 hr fluctuations observed in $A L$ and PCN indices. Post onset, the $A U$ index reached similar levels during sawtooth events and shock initiated onsets.

- Ring current intensity as measured by the SYM$\mathrm{H}$ was found to be around the storm levels during sawtooth events throughout the epoch window with similar quasi-periodic fluctuations observed in the partial recovery of the index. On average, the ASYM-H response to quasi-periodic flux injections at geosynchronous orbit was delayed by around 40 mins compared to fluctuations in the SYM-H index.

- In both the event groups, the latitudinal thickness of the oval in the noon sector did not show any significant variation during the epoch window due to both the boundaries moving equatorward before the onset and poleward after the onset. It was also seen that the oval thickness was around the same during both the event groups. The dawn sector experienced a clear thinning and widening of the oval around the onset during both sawtooth events and shock related onsets. This sequence was however, not observed in the dusk sector and could point to a stronger substorm activity in the dawn MLTs compared to dusk MLTs [Mende et al. 2003].

- Sawteeth events were characterized by large variations of the magnetic field at the geosynchronous orbit. The tail field strength became weak compared to shock related onsets due to magnetotail stretching and rapidly relaxed to much stronger values after the onset.

Acknowledgements. The author wishes to acknowledge CDAWeb for the solar wind and geomagnetic activity data. This research was supported by the Dr. D. S. Kothari post doctoral fellowship (EN/14-15/0025).

\section{References}

Abraham-Shrauner, B. (1972). Determination of magnetohydrodynamic shock normals. Journal of Geophysical Research, 77(4), 736-739. https:// doi.org/10.1029/JA077i004p00736

Akasofu, S.-I. (1981). Energy coupling between the solar wind and the magnetosphere. Space Science Reviews, 28(2), 121-190. https:/ / doi.org/10.1007/BF00218810

Akasofu, S.-I., \& Chao, J. K. (1980). Interplanetary shock waves and magnetospheric substorms. Planetary and Space Science, 28, 381-385. https:/ / doi.org/10.1016/0032-0633(80)90042-2

Baker, D. N., Pulkkinen, T. I., Angelopoulos, V., Baumjohann, W., \& McPherron, R. L. (1996). Neutral line model of substorms: Past results and present view. Journal of Geophysical Research: Space Physics, 101(A6), 12975-13010. https: / / doi.org/10.1029/95JA03753

Baker, K. B., \& Wing, S. (1989). A new magnetic coordinate system for conjugate studies at high latitudes. Journal of Geophysical Research: Space Physics, 94(A7), 9139-9143. https:// doi.org/10.1029/JA094iA07p09139

Belian, R. $\sim$ D., Cayton, T. $\sim$ E., \& Reeves, G. $\sim$ D. (1995). Quasi-Periodic Global Substorm Generated Flux Variations Observed at Geosynchronous Orbit. Washington DC American Geophysical Union Geophysical Monograph Series, 86, 143.

Brittnacher, M., Wilber, M., Fillingim, M., Chua, D., Parks, G., Spann, J., \& Germany, G. (2000). Global auroral response to a solar wind pressure pulse. Advances in Space Research, 25(7), 1377-1385. https: / / doi.org/http: / / dx.doi.org/10.1016/S02731177(99)00647-X

Curto, J. J., Araki, T., \& Alberca, L. F. (2007). Evolution of the concept of Sudden Storm Commencements and their operative identification. Earth, Planets and Space, 59(11), i-xii. https:/ / doi.org/10.1186/BF03352059

Frey, H. U., Mende, S. B., Angelopoulos, V., \& Donovan, E. F. (2004). Substorm Onset Observations by IMAGEFUV. Journal of Geophysical Research, 109.

Gosling, J. T., \& Pizzo, V. J. (1999). Formation and Evolution of Corotating Interaction Regions and their Three Dimensional Structure. Space Science Reviews, 89(1), 21-52. https:/ / doi.org/10.1023/A:1005291711900

Grocott, A., Wild, J. A., Milan, S. E., \& Yeoman, T. K. (2009). Superposed epoch analysis of the ionospheric convection evolution during substorms: onset latitude dependence. Annales Geophysicae, 
27(2), 591-600. https: / doi.org/10.5194/ angeo-27591-2009

Henderson, M. G., Skoug, R., Donovan, E., Thomsen, M. $\sim$ F., Reeves, G. $\sim$ D., Denton, M. $\sim$ H., ... Frank, L. $\sim$ A. (2006). Substorms during the 10-11 August 2000 sawtooth event. Journal of Geophysical Research (Space Physics), 111, A06206. https: / / doi.org/10.1029/2005JA011366

Henderson, M. G., Reeves, G. D., Skoug, R., Thomsen, M. F., Denton, M. H., Mende, S. B., ... Singer, H. J. (2006). Magnetospheric and auroral activity during the 18 April 2002 sawtooth event. Journal of Geophysical Research: Space Physics, 111(A1), n/a--n/a. https: / / doi.org/10.1029/2005JA011111

Kallio, E. I., Pulkkinen, T. I., Koskinen, H. E. J., Viljanen, A., Slavin, J. A., \& Ogilvie, K. (2000). Loadingunloading processes in the nightside ionosphere. Geophysical Research Letters, 27(11), 1627-1630. https: / / doi.org/ 10.1029/ 1999GL003694

Kamide, Y., \& Kokubun, S. (1996). Two-component auroral electrojet: Importance for substorm studies. Journal of Geophysical Research: Space Physics, 101(A6), 13027-13046.

https: / / doi.org/10.1029/96JA00142

Kamide, Y., \& Rostoker, G. (2004). What is the physical meaning of the AE index? Eos, Transactions American Geophysical Union, 85(19), 188-192. https: / / doi.org/10.1029/2004EO190010

King, J. H., \& Papitashvili, N. E. (2005). Solar wind spatial scales in and comparisons of hourly Wind and ACE plasma and magnetic field data. Journal of Geophysical Research: Space Physics, 110(A2), A02104. https: / / doi.org/10.1029/ 2004JA010649

Kokubun, S., McPherron, R. L., \& Russell, C. T. (1977). Triggering of substorms by solar wind discontinuities. Journal of Geophysical Research, 82(1), 74-86. https: / / doi.org/10.1029/JA082i001p00074

Kozelova, T. V, \& Kozelov, B. V. (2013). Substorm-associated explosive magnetic field stretching near the earthward edge of the plasma sheet. Journal of Geophysical Research: Space Physics, 118(6), 33233335. https: / / doi.org/10.1002/jgra.50344

Liou, K., Newell, P. T., Meng, C.-I., Brittnacher, M., \& Parks, G. (1998). Characteristics of the solar wind controlled auroral emissions. Journal of Geophysical Research: Space Physics, 103(A8), 17543-17557. https: / / doi.org/10.1029/98JA01388

Longden, N., Chisham, G., Freeman, M. P., Abel, G. A., \& Sotirelis, T. (2010). Estimating the location of the open-closed magnetic field line boundary from auroral images. Annales Geophysicae, 28(9), 16591678. https: / / doi.org/10.5194/ angeo-28-1659-2010
McComas, D. J., Bame, S. J., Barker, P., Feldman, W. C., Phillips, J. L., Riley, P., \& Griffee, J. W. (1998). Solar Wind Electron Proton Alpha Monitor (SWEPAM) for the Advanced Composition Explorer. Space Science Reviews, 86(1), 563-612.

https: / / doi.org/ 10.1023/ A:1005040232597

McPherron, R. L., Terasawa, T., \& Nishida, A. (1986). Solar wind triggering of substorm expansion onset. Journal of Geomagnetism and Geoelectricity, 38, 1089-1108.

Mende, S. B., Frey, H. U., Morsony, B. J., \& Immel, T. J. (2003). Statistical behavior of proton and electron auroras during substorms. Journal of Geophysical Research, 108.

Meurant, M., Gérard, J.-C., Blockx, C., Coumans, V., Hubert, B., Connors, M., ... Donovan, E. (2005). Comparison of intense nightside shock-induced precipitation and substorm activity. Journal of Geophysical Research: Space Physics, 110(A7), A07228. https: / / doi.org/10.1029/2004JA010916

Oliveira, D. M. (2017). Magnetohydrodynamic Shocks in the Interplanetary Space: a Theoretical Review. Brazilian Journal of Physics, 47(1), 81-95. https: / / doi.org/10.1007/ s13538-016-0472-x

Oliveira, D. M., \& Raeder, J. (2014). Impact angle control of interplanetary shock geoeffectiveness. Journal of Geophysical Research: Space Physics, 119(10), 8188-8201. https: / / doi.org/10.1002/2014JA020275

Partamies, N., Pulkkinen, T. I., McPherron, R. L., McWilliams, K., Bryant, C. R., Tanskanen, E., ... Thomsen, M. F. (2009). Statistical survey on sawtooth events, SMCs and isolated substorms. Advances in Space Research, 44(3), 376-384. https: / / doi.org/http: / / dx.doi.org/10.1016/j.asr.20 09.03.013

Pulkkinen, T. I., Ganushkina, N. Y., Tanskanen, E. I., Kubyshkina, M., Reeves, G. D., Thomsen, M. F., ... Gjerloev, J. (2006). Magnetospheric current systems during stormtime sawtooth events. Journal of Geophysical Research: Space Physics, 111(A11), n/a-n/a. https: / / doi.org/10.1029/2006JA011627

Pulkkinen, T. I., Partamies, N., McPherron, R. L., Henderson, M., Reeves, G. D., Thomsen, M. F., \& Singer, H. J. (2007). Comparative statistical analysis of storm time activations and sawtooth events. Journal of Geophysical Research: Space Physics, 112(A1), n/a--n/a. https: / / doi.org/10.1029/2006JA012024

Sandholt, P. E., Farrugia, C. J., \& Denig, W. F. (2014). MI coupling across the auroral oval at dusk and midnight: repetitive substorm activity driven by interplanetary coronal mass ejections (CMEs). An- 
nales Geophysicae, 32(4), 333-351. https: / / doi.org/10.5194/ angeo-32-333-2014

Singer, H., Matheson, L., Grubb, R., Newman, A., \& Bouwer, D. (1996). Monitoring space weather with the GOES magnetometers. In E. $\sim$ R. Washwell (Ed.), GOES-8 and Beyond (Vol. 2812, pp. 299-308). https: / / doi.org/ 10.1117/ 12.254077

Smith, C. W., L’Heureux, J., Ness, N. F., Acuña, M. H., Burlaga, L. F., \& Scheifele, J. (1998). The ACE Magnetic Fields Experiment. Space Science Reviews, 86(1), 613-632.

https: / / doi.org/10.1023/ A:1005092216668

Tanskanen, E., Pulkkinen, T. I., Koskinen, H. E. J., \& Slavin, J. A. (2002). Substorm energy budget during low and high solar activity: 1997 and 1999 compared. Journal of Geophysical Research: Space Physics, 107(A6), SMP 15-1--SMP 15-11. https: / / doi.org/10.1029/2001JA900153

Tsurutani, B. T., \& Lin, R. P. (1985). Acceleration of $>47 \mathrm{keV}$ Ions and $>2 \mathrm{keV}$ electrons by interplanetary shocks at 1 AU. Journal of Geophysical Research: Space Physics, 90(A1), 1-11. https: / / doi.org/10.1029/JA090iA01p00001

Yao, L., Liu, Z., Zuo, P., Zhang, L., \& Duan, S. (2009). Responses of properties in the plasma sheet and at the geosynchronous orbit to interplanetary shock. Chinese Science Bulletin, 54(18), 3308-3317. https: / / doi.org/10.1007/s11434-009-0213-4

Zhou, X., \& Tsurutani, B. T. (2001). Interplanetary shock triggering of nightside geomagnetic activity: Substorms, pseudobreakups, and quiescent events. Journal of Geophysical Research: Space Physics, 106(A9), 18957-18967.

https://doi.org/10.1029/2000JA003028

doi:10.1002/2013EO450001.

\footnotetext{
${ }^{*}$ Corresponding author: Tushar Andriyas,

Nehru Science Center, University of Allahabad, India;

email: tushar.andriyas@aggiemail.usu.edu

C 2017 by the Istituto Nazionale di Geofisica e Vulcanologia. All rights reserved.
} 